Psychotherapeut 2009 $54: 409-410$

DOI 10.1007/s00278-009-0708-0

Online publiziert: 28. Oktober 2009

(c) Springer Medizin Verlag 2009
Bernhard Strauß $\beta^{1}$ Harald J. Freyberger ${ }^{2}$

${ }^{1}$ Institut für Psychosoziale Medizin und Psychotherapie, Universitätsklinikum, Friedrich-Schiller-Universität Jena,

${ }^{2}$ Klinik für Psychiatrie und Psychotherapie, Universitätsklinikum Greifswald

\title{
Ausbildungsforschung in der Psychotherapie
}

\section{Ergebnisse des Forschungsgutachtens}

Die Ausbildung künftiger Psychotherapeuten ist seit Langem ein florierender Markt. Insbesondere nach Inkrafttreten des so genannten Psychotherapeutengesetzes, dessen zehnten Geburtstag wir in diesem Jahr feiern konnten, hat sich die Ausbildung psychologischer Psychotherapeuten sowie Kinder- $u$. Jugendlichenpsychotherapeuten neu formiert und geordnet. Der großen Bedeutung der Ausbildung innerhalb des Feldes steht gegenüber, dass schon immer vergleichsweise geringe Forschungsbemühungen auf diesen Bereich gerichtet waren. Dies mag auch damit zu tun haben, dass die Person des Psychotherapeuten als Gegenstand von Psychotherapieforschung ohnehin erst in jüngster Zeit und noch sehr zögerlich berücksichtigt wird und dementsprechend auch die Ausbildung von Psychotherapeuten lange eine untergeordnete Bedeutung hatte.

Es versteht sich, dass aus wissenschaftlichen, berufspolitischen, ethischen Gründen und im Interesse einer stetigen Verbesserung der Qualität psychotherapeutischer Maßnahmen die Ausbildung eine ganz zentrale Rolle spielt und eigentlich sehr viel mehr Forschungsbemühungen verdienen würde. Aus diesem Grund haben sich die Herausgeber des Psychotherapeut entschlossen, passend zum zehnjährigen Jubiläum des Psychotherapeutengesetzes, ein Schwerpunktheft zum Thema Ausbildungsforschung zu gestalten. Ein weiterer Grund war das durch das Bundesministerium für Gesundheit im Jahr 2007 ausgeschriebene und 2009 abgeschlossene Forschungsgutachten zur Ausbildung psychologischer Psychotherapeuten und Kinder- und Jugendlichenpsychotherapeuten (Strauß et al. 2009), das eine mögliche Grundlage für die Novellierung des Psychotherapeutengesetzes auf der Basis empirischer Untersuchungen und intensiver Bewertungen des Standes der psychotherapeutischen Ausbildung in Deutschland und im Ausland liefern sollte.

Im Rahmen des Forschungsgutachtens wurde eine ganze Reihe von Befragungen durchgeführt, beispielsweise von Studierenden, Aufsichtsbehörden, Ausbildungsteilnehmern, Absolventen der Ausbildung, Lehrkräften, Praxiseinrichtungen und Ausbildungsstätten, wodurch eine Fülle an informativem Material zusammengestellt werden konnte, das eine sehr differenzierte Bewertung der Stärken und Schwächen der aktuellen Ausbildung zulässt. In drei Beiträgen des Heftes (Sonntag et al., Glaesmer et al., Kohl et al.) stellen wir einige Kernergebnisse der Befragungen von Teilnehmern, Absolventen und Lehrkräften dar. Ein weiterer Originalbeitrag widmet sich der Frage, wie unterschiedlich bzw. ähnlich Psychotherapieausbildung sowie die Verankerung der Psychotherapie im Gesundheitswesen im Vergleich europäischer Länder zu sehen sind. Im Zuge einer fortschreitenden Europäisierung des Gesundheitswesens ist es sicherlich bedeutsam, einen Blick über die Grenzen zu wagen, um möglicherweise auch zu antizipieren, welche Veränderungen durch eine potenzielle europawei- te Normierung auf die Psychotherapie zukommen können.

In der Übersichtsarbeit dieses Heftes haben wir (Strauß u. Kohl) versucht, zumindest kursorisch einen Überblick über den Stand der Ausbildungsforschung zu geben und ein Modell zu präsentieren, anhand dessen die vielen Forschungsbefunde, die es bereits gibt und die hoffentlich in Zukunft gesammelt werden, zu organisieren bzw. zu ordnen. Die Übersicht macht auch deutlich, wo die Ausbildungsforschung bisher noch Defizite hat und welche Bereiche künftig vermehrt untersucht werden sollten.

\section{Zurück zum Forschungsgutachten}

In dem Beitrag der Rubrik „Psychotherapie aktuell“" wird gezeigt, dass es zum Zeitpunkt der Arbeit an dem Forschungsgutachten ungefähr 18o Ausbildungsinstitute in der Bundesrepublik gab, die sich im Wesentlichen auf die Vertiefungsverfahren richten, die auch als „Richtlinienverfahren“ gelten. Dies wird sich durch die Empfehlung der systemischen Therapie als Verfahren für die vertiefte Ausbildung durch den Wissenschaftlichen Beirat möglicherweise in nächster Zeit noch sehr verändern. Die im Psychotherapeutengesetz festgelegten Rahmenbedingungen für die Ausbildung haben sich zu großen Teilen bewährt, sind aber durchaus noch reformbedürftig. Das Forschungsgutachten bezog sich ausschließlich auf die Ausbildung psychologischer Psychotherapeuten und Kinder- und Jugendlichenpsychothe- 
rapeuten und betrachtete nicht die Weiterbildung von Ärzten im Bereich der Psychotherapie, die sicherlich einer ähnlichen Bestandsaufnahme bedarf.

Vor dem Hintergrund der zahlreichen Befunde, die im Forschungsgutachten erhoben wurden, einer intensiven Analyse von universitären sowie postgradualen Ausbildungsgängen und verschiedener Expertenbefragungen hat das Forschungsgutachten eine Reihe von Bewertungen vorgenommen, die dem Gesetzgeber dazu dienen könnten, eine Reform der Ausbildung zu initiieren.

Letztendlich kommt das Gutachten zu dem Schluss, dass eine grundlegende Veränderung der Ausbildungsstruktur nicht sinnvoll ist, dass aber beispielsweise die strikte Verfahrensorientierung der Ausbildung durch eine intensivere Vermittlung von Inhalten anderer Verfahren und ggf. Methoden aufgelockert werden sollte. Im Zuge der so genannten Bologna-Reform haben sich die Studiengänge für Psychologen und Pädagogen grundsätzlich geändert, was ein Anlass für die Vergabe des Gutachtens war. Das Gutachten spricht sich eindeutig dafür aus, den Master-Abschluss als Basis für die Zulassung zu einer Psychotherapieausbildung zu fordern und gleichzeitig bestimmte Inhalte, insbesondere aus dem Fachgebiet der Psychologie, als notwendige Voraussetzung für die $\mathrm{Zu}$ lassung zur Ausbildung festzulegen.

Das Gutachten spricht sich außerdem dafür aus, ein geändertes Modell für die Ausbildung von Psychotherapeuten, die entweder mit Erwachsenen oder mit Kindern und Jugendlichen arbeiten, zu etablieren und postuliert die Einrichtung eines „common trunk“, auf dessen Basis grundsätzlich eine Doppelapprobation möglich werden kann und die dazu führt, dass es zukünftig Psychotherapeuten gibt, die im „Schwerpunkt Erwachsene" und/ oder im „Schwerpunkt Kinder- u. Jugendliche“ ausgebildet sind.

Nach einer sorgfältigen Prüfung von Alternativen kommt das Gutachten zu dem Schluss, dass an einer - wenngleich modifizierten - „Ausbildung nach der Ausbildung“ festgehalten werden sollte. Theoretische Unterrichtsinhalte könnten zum Teil aber sehr viel mehr in die grundständigen Studiengänge verlagert werden, was der praktischen Ausbildung und der
Vermittlung vertiefender Inhalte mehr Raum geben würde. Das Gutachten empfiehlt aber durchaus auch die Einrichtung von evaluierten Modellstudiengängen, die eine „Direktausbildung“ erproben könnten.

Ein kritisches Thema, um das sich das Gutachten rankt, bezieht sich auf die Ausbildungskosten im engeren und weiteren Sinne, insbesondere auf die mögliche Vergütung der praktischen Tätigkeit. Hier wurde der Gesetzgeber gebeten, dringend Änderungen zu initiieren, zu denen auch neue Modelle der Ausbildungsförderung gehören sollten.

Im Zusammenhang mit der - speziell in medizinischen Fachverbänden besonders kritisch diskutierten - Frage der Medizinorientierung in der psychologischen Psychotherapie wurde empfohlen - nicht zuletzt im Hinblick auf die Versorgungsqualität - Psychotherapeuten die Möglichkeit zu geben, künftig auch Arbeitsunfähigkeitsbescheinigungen auszustellen, zu Fachärzten oder in eine psychiatrische bzw. psychosomatische Klinik über- bzw. einzuweisen sowie psychotherapiespezifische Heil- und Hilfsmittel zu verschreiben. Das Gutachten sprach sich allerdings dagegen aus, nichtärztlichen Psychotherapeuten die Möglichkeit der Medikamentenverschreibung einzuräumen.

Schließlich unterbreitet das Gutachten ganz spezifische Vorschläge für die inhaltliche und organisatorische Gestaltung der Ausbildung, zu der eine deutliche Verkürzung der Gesamtstundenzahl (von bisher 4200 Stunden auf 3400 Stunden) gehört. Insbesondere durch eine Reduktion der so genannten freien Verfügungsstunden, aber auch eine stringentere und kompaktere Planung der praktischen Tätigkeit, die sich insgesamt auf nunmehr 1200 Stunden reduzieren sollte, kann diese Gesamtkürzung sehr gut erreicht werden und dazu beitragen, dass die Ausbildungsteilnehmer in der zur Verfügung stehenden Zeit die Ausbildung abschließen können, was zurzeit für Viele nicht möglich ist. In den Vorschlägen sind auch Wünsche der Ausbildungsteilnehmer berücksichtigt, beispielsweise die Ausweitung der Anteile von Supervision und Selbsterfahrung bzw. die Kombination von Einzelund Gruppen-Settings in der Supervision und Selbsterfahrung, die bislang nur teilweise gegeben ist.

Wir hoffen, dass das Forschungsgutachten und die dadurch vielleicht ausgelösten Diskussionen dazu beitragen können, die aktuelle Situation der Ausbildung von Psychotherapeuten zu verbessern sowie die Qualität und Qualitätssicherung zu erhöhen, die teilweise - auch das war ein Befund des Gutachtens - sicher noch im Argen liegt.

Wir hoffen außerdem, dass die einzelnen Studien, die im Rahmen des Gutachtenverfahrens durchgeführt wurden, dazu beitragen können, dass die Ausbildungsforschung neue Impulse erhält und Psychotherapieforscher an diesem Bereich mehr Interesse finden. Es gibt einige Zeichen, die dies andeuten, zum Beispiel die Formierung von Interessengruppen innerhalb der Society for Psychotherapy Research.

\section{Korrespondenzadresse \\ Prof. Dr. Bernhard Strauß}

Institut für Psychosoziale Medizin und Psychotherapie, Universitätsklinikum, Friedrich-Schiller-Universität Jena

Stoystr. 3, 07740 Jena

Bernhard.Strauss@med.uni-jena.de

Danksagung. Abschließend wollen wir die Gelegenheit nutzen, auch an dieser Stelle noch einmal dem Bundesministerium für Gesundheit (BMG) und allen Personen herzlich zu danken, die zum Zustandekommen des Forschungsgutachtens beigetragen haben. Zu diesem Personenkreis gehören auch alle Autoren dieses Heftes, denen für ihre Beiträge ebenfalls Dank gebührt.

Interessenkonflikt. Der korrespondierende Autor gibt an, dass kein Interessenkonflikt besteht.

\section{Literatur}

Strauß B, Barnow S, Brähler E et al. (2009) Forschungsgutachten zur Ausbildung von Psychologischen Psychotherapeuten und Kinder- und Jugendlichenpsychotherapeuten. http://www.med.unijena.de/mpsy/forschungsgutachten/Endfassung_ Forschungsgutachten_Psychotherapieausbildung.pdf 